\title{
Numerical simulations and experiments of simultaneous acceleration of positive and negative ions in a radio frequency quadrupole
}

\author{
X. Q. Yan, * Y. R. Lu, J. X. Fang, Z. Y. Guo, X. T. Ren, J. X. Yu, Y. Xie, and C. E. Chen \\ MOE Key Laboratory of Heavy Ion Physics, Peking University, Beijing, 100871, China
}

(Received 6 October 2004; published 1 February 2006)

\begin{abstract}
The simultaneous acceleration of $\mathrm{O}^{+}$and $\mathrm{O}^{-}$beams in a radio frequency quadruple accelerator has been numerically simulated and the results are presented in this paper. The micropulses of $\mathrm{O}^{+}$and $\mathrm{O}^{-}$ beams have been measured in dual beam acceleration experiments, which verified the numerical simulations and feasibilities of dual beam acceleration.
\end{abstract}

DOI: 10.1103/PhysRevSTAB.9.020101

PACS numbers: 29.27. - a, 29.17.+w, 29.27.Bd

\section{INTRODUCTION}

A continuous dc beam can be gradually bunched into a series of buckets in radio frequency quadrupole (RFQ) accelerators. For positive and negative ions, there are two buckets in every rf period, respectively. If both ions are mixed and simultaneously injected into the RFQ, they can be bunched into their own buckets $180^{\circ}$ apart in the same rf period. When positive and negative ions are homogeneously mixed, neutralization of space charge is automatically realized in the region between the bending magnet for beam mixing and the bunching section of the RFQ; then the emittance growth of the mixed beams is restrained, because the space charge fields are weakened by full or partial charge neutralization. However, as the continuous beam begins to be bunched and accelerated, the ions with a different sign of charge exert forces that resist the separation of the ions, so the transmission efficiency is not improved compared with single beam acceleration [1-3]. However, it is still attractive in some applications that the total output beam flux can be easily doubled by simultaneous acceleration of ions injected from a positive and a negative ion source. The integral split ring (ISR) RFQs have been developed at Peking University since the 1980s in collaboration with the Institute of Applied Physics (IAP) at Frankfurt University [4]; up to now two ISR RFQs have been commissioned $[5,6]$. To investigate the feasibilities and advantages of the simultaneous acceleration of positive and negative oxygen ions in an ISR RFQ, numerical simulations and dual beams acceleration experiments have been carried out.

\section{ISR 1000}

The principal parameters of the ISR 1000 RFQ are listed in Table I. The beam dynamics simulations were done with the well-known LANL software PARMTEQ [7] and Fig. 1 shows the RFQ parameters along the structures, where pal and pat are abbreviations for phase advance longitudinal

\footnotetext{
*Corresponding author.

Email address: X.yan@pku.edu.cn
}

and phase advance transverse; $a, w, \Phi \mathrm{s}$, and $m$ are aperture, energy, synchronous phase, and electrode modulation, respectively. The minivane electrodes [8] and the stem geometries are shown in Fig. 2.

\section{SIMULATIONS FOR DUAL BEAMS ACCELERATION}

The single beam dynamics simulations of RFQ-1000 were initially done with the well-known RFQ codePARMTEQ. The longitudinal dynamics simulation is given in Fig. 3, where the positive ions are homogeneously distributed in the range of $[-3 \pi / 2, \pi / 2]$. Main parameters of the simulation are listed in Table II.

To simulate the dual beams acceleration, LANL code PARMTEQ was modified as DYNRFQ [9], where an array was introduced to record charge property for every particle. After the positive and negative ions are mixed and injected into the RFQ, both kinds of ions are bunched, respectively, into their own buckets. Meanwhile the repulsive and attractive fields of space charge are separately determined by the SCHEFF subroutine, which uses the two-dimensional $(r-z)$ PIC method [10].

The parameters for dual beams acceleration are listed in Table II. The longitudinal dynamics simulation for dual beams is illustrated in Fig. 4. At first the positive ions are homogeneously distributed in the range of [ $-3 \pi / 2, \pi / 2]$ while the negative ions are uniformly distributed in the range of $[-5 \pi / 2,-\pi / 2]$, then two kinds of ions are

TABLE I. Principal parameters of ISR RFQ-1000.

\begin{tabular}{lc}
\hline \hline & ISR RFQ-1000 \\
\hline Ion species & $\mathrm{O}^{+}, \mathrm{O}^{-}, \mathrm{N}^{+}$ \\
$f(\mathrm{MHz})$ & 26 \\
$W_{\text {in }}(\mathrm{keV})$ & 22 \\
$W_{\text {out }}(\mathrm{keV})$ & 1000 \\
Length $(\mathrm{cm})$ & 260 \\
Diameter $(\mathrm{cm})$ & 75 \\
$V_{0}(\mathrm{kV})$ & 70 \\
Duty factor & $1 \mathrm{~ms} / 6 \mathrm{~ms}$ \\
\hline \hline
\end{tabular}




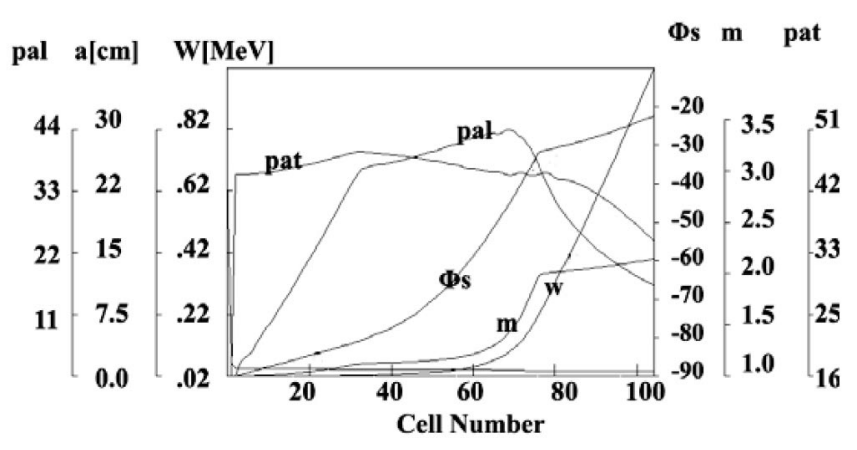

FIG. 1. Dynamics design of ISR RFQ-1000.

separated and bunched gradually to their own synchronous phase. Figure 5 illustrates the bunching process in the longitudinal phase space for $\mathrm{O}^{+}$and $\mathrm{O}^{-}$beams.

The results of both simulations are given also in Table II. It shows that the transmission efficiency is a little decreased and the energy spread and emittance growth are limited while the beam current is $5 \mathrm{~mA}\left(\mathrm{O}^{+} 5 \mathrm{~mA}\right.$ and $\mathrm{O}^{-}$ $5 \mathrm{~mA}$ ), compared with single beam acceleration. So it is possible that the output beam flux can be easily increased

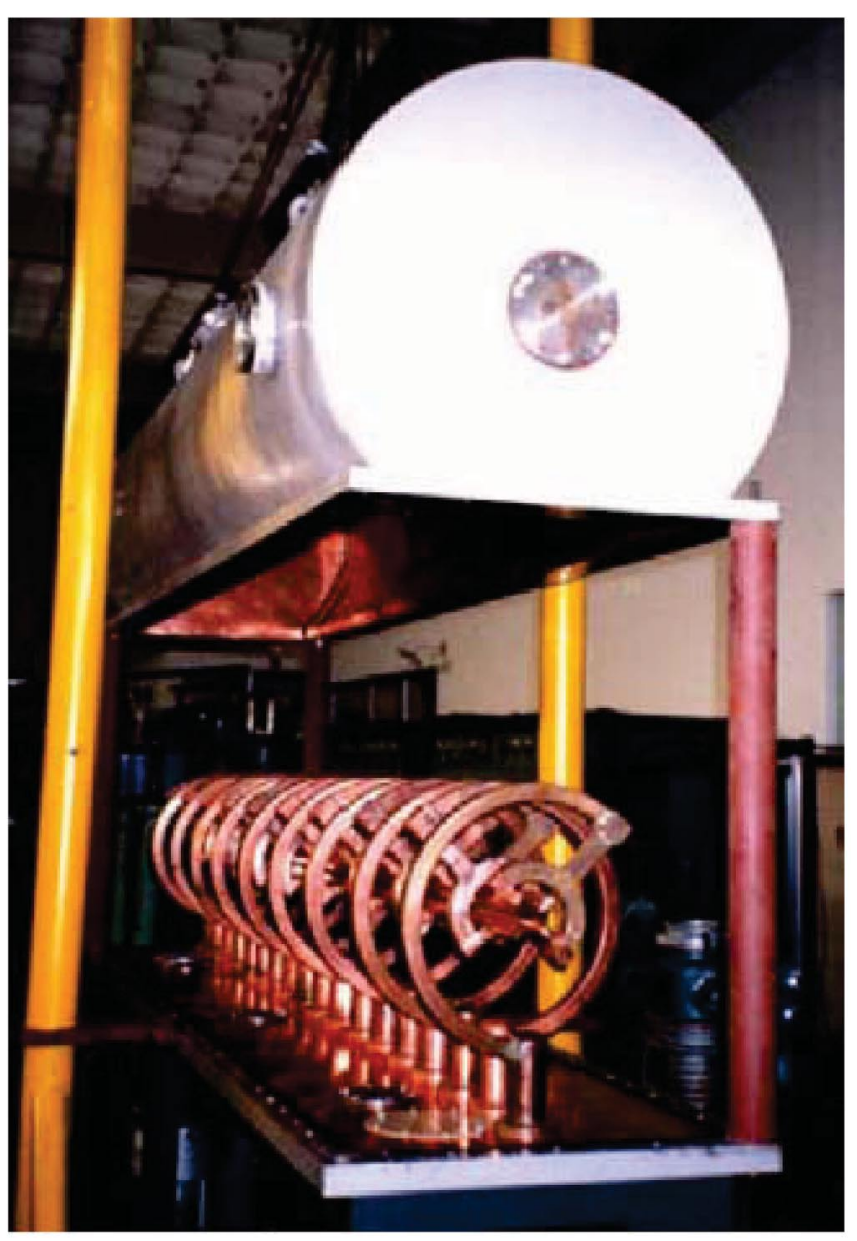

FIG. 2. (Color) Photo of ISR RFQ-1000.

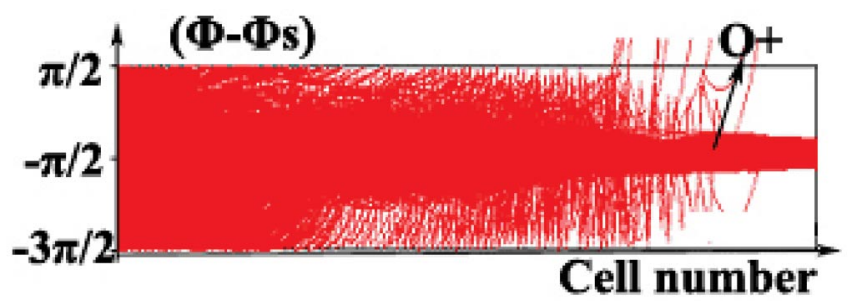

FIG. 3. (Color) Longitudinal dynamics simulation for single beam $\left(\Phi-\Phi_{\mathrm{s}}\right.$ is the phase difference between a particle and the synchronous particle).

TABLE II. Main parameters of simulations.

\begin{tabular}{lcc}
\hline \hline & Single beam & Dual beams \\
\hline Number of $\mathrm{O}^{+}$ & 5000 & 2500 \\
Number of $\mathrm{O}^{-}$ & 0 & 2500 \\
${\text { Current of } \mathrm{O}^{+}(\mathrm{mA})}$ & 5 & 5 \\
Current of $\mathrm{O}^{-}(\mathrm{mA})$ & 0 & 5 \\
Phase width & $2 \pi$ & $2 \pi$ \\
$\varepsilon_{\text {xin }}(\mathrm{mm}$ mrad, RMS, norm) & 0.2 & 0.2 \\
$\varepsilon_{\text {out }}(\mathrm{mm}$ mrad, RMS, norm) & 0.358 & 0.548 \\
Energy spread (entrance) & 0 & 0 \\
Energy spread (exit) & $1 \%$ & $4 \%$ \\
Transmission (\%) & 96.3 & 92.1 \\
\hline \hline
\end{tabular}

by $90 \%$ in a simultaneous acceleration of ions, which are injected from a positive and a negative ion source in a modest beam current $(5 \mathrm{~mA})$. As long as the current augments, the transmission efficiency for dual beams is decreased more quickly than in the case of the single beam because of stronger mutual interactions between the two species of beams, which means a special design strategy is necessary for dual beams acceleration to obtain higher transmission efficiency. The efficiencies are plotted in Fig. 6 for single beam and dual beams acceleration, respectively.

\section{THE EXPERIMENT OF DUAL BEAMS ACCELERATION FOR ISR RFQ-1000}

Figure 7 shows the layout of ISR RFQ-1000 beam transport line. Two permanent magnetic PIG ion sources

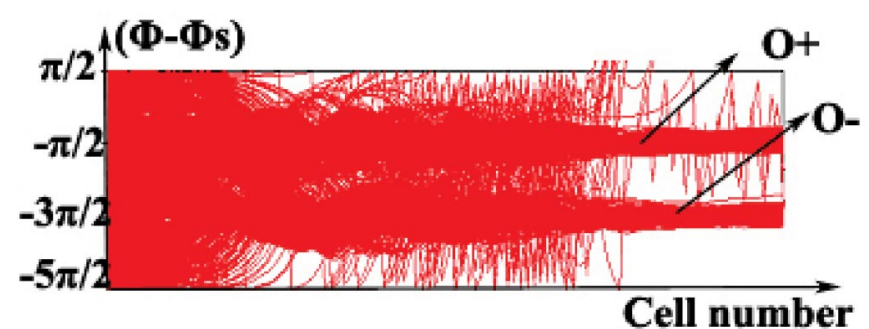

FIG. 4. (Color) Longitudinal dynamics simulation for dual beams. 


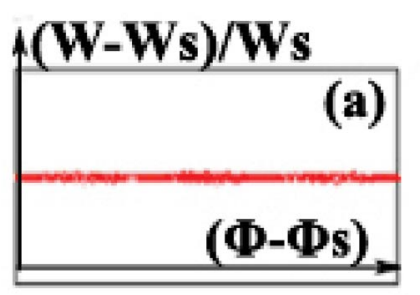

(W-Ws)/Ws
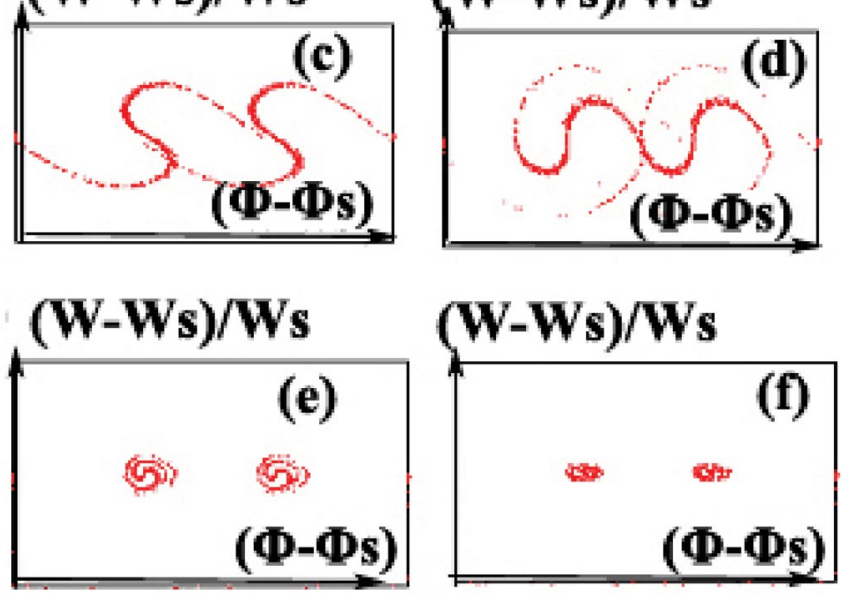

FIG. 5. (Color) Process of bunching in the simultaneous acceleration of positive and negative ions.

are located at $45^{\circ}$ to the beam axis. One is a side extraction PIG ion source with cold cathode; another is a pocket end extraction sputtering PIG ion source. Both positive and negative oxygen beams can be bent and combined together by the funneling magnet (FM). Then the beams are focused by an Einzel lens (EL) and injected to the RFQ accelerator.

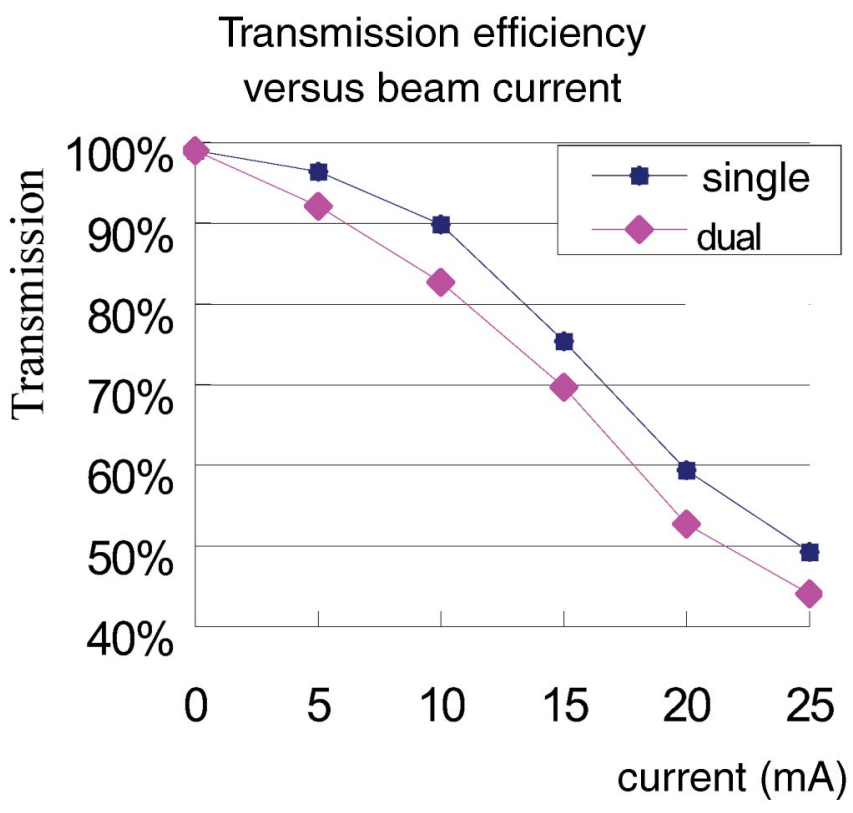

FIG. 6. (Color) Transmission efficiency versus beam current.

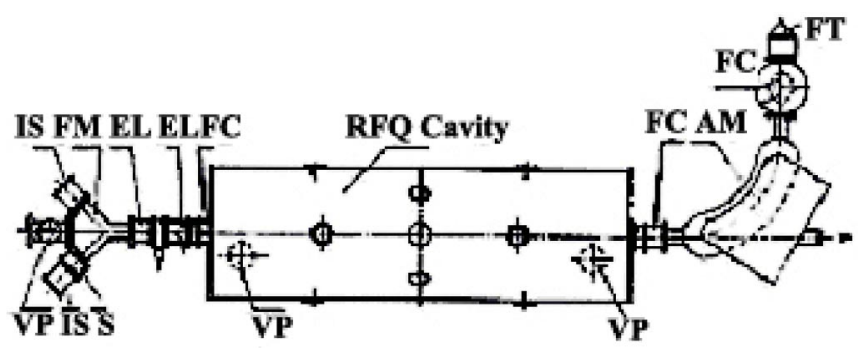

FIG. 7. Layout of ISR RFQ-1000 beam transport line.

Figure 8 shows the time structure of the micropulse of the $\mathrm{O}^{+}$beam only, while Fig. 9 is for the simultaneous acceleration of both $\mathrm{O}^{+}$and $\mathrm{O}^{-}$beams. Its time structure was measured by a coaxial fast Faraday cup. The output beams hit on the coaxial inner conductor or beam absorber, which was connected to a very broadband cone $50 \Omega$ transmission line. Then a fast amplifier amplifies the signal generated by the beams, which is displayed on a fast oscilloscope triggered by rf pickup signal of the RFQ cavity. The accelerated positive and negative oxygen beams in Fig. 9 are bunched clearly to their own buckets and the synchronous phase difference is $\pi$, which corresponds to Figs. 4 and 5. The absolute beam synchronous phase and the micropulse beam intensity were not yet calibrated.

\section{CONCLUSION}

In this paper simultaneous injection of $\mathrm{O}^{-}$and $\mathrm{O}^{+}$ beams into a $26 \mathrm{MHz}$ RFQ was numerically simulated. The results showed that although the transmission was a little decreased, the energy spread and the emittance growth were also increased over that in single beam accel-

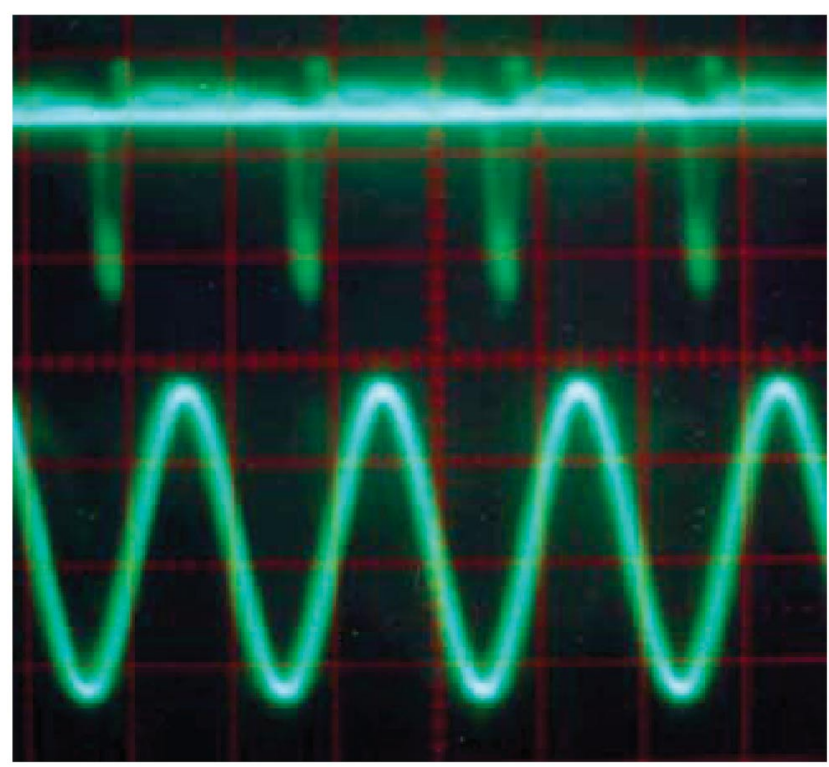

FIG. 8. (Color) Micropulse time structure of $\mathrm{O}^{+}$beam. 


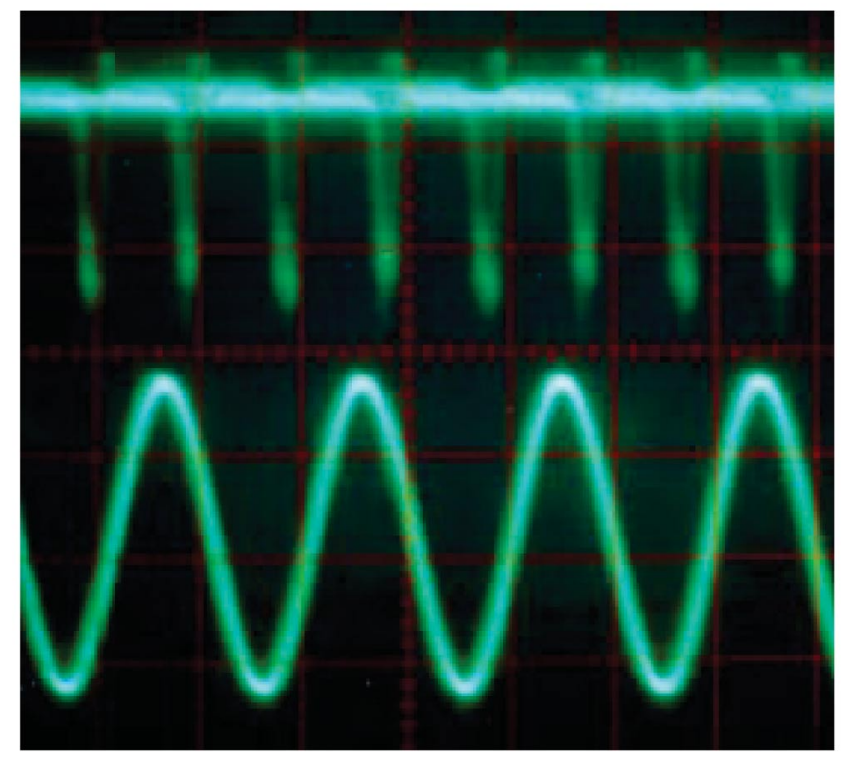

FIG. 9. (Color) Micropulse time structure of $\mathrm{O}^{-}$and $\mathrm{O}^{+}$beam.

eration; however, the total output beam flux can be increased in the simultaneous acceleration of positive and negative ions, as each macropulse has nearly 2 times as many ions when the beam current is modest. Moreover, little or no accumulation of charges is left on the target when ISR RFQ 1000 is used for the ion implantation, which is advantageous for the production of the new semiconductor material or components. Furthermore the dual beam acceleration was carried out in the ISR RFQ 1000 and the micropulse time structures of beams were clearly observed in the experiment, verifying the numerical simulations and feasibilities of dual beams acceleration.

\section{ACKNOWLEDGMENTS}

This work was supported by NSFC, China (Contract No. 10455001).

[1] Y. Oguri, Nucl. Instrum. Methods Phys. Res., Sect. A 373, 175 (1996).

[2] K.R. Crandall, in Proceedings of the IEEE Particle Accelerator Conference, San Francisco, CA, 1991 (IEEE, Piscataway, NJ, 1991), p. 401.

[3] J.X. Yu et al., At. Energ. Sci. Technol. 35, 37 (2001).

[4] J.X. Fang and C. E. Chen, IEEE Trans. Nucl. Sci. 32, 2981 (1985).

[5] C. E. Chen et al., in Proceedings of the European Particle Accelerator Conference, Berlin 1992 (Frontíeres, Gif-surYvette, 1992), p. 1328.

[6] Y. Lu et al., in Proceedings of the European Particle Accelerator Conference, Vienna, 2000 (EPS, Geneva, 2000).

[7] K.R. Crandall et al., in Proceedings of the Linear Accelerator Conference, 1979 (Brookhaven National Laboratory, Upton, NY, 1980), p. 205.

[8] Y. R. Lu et al., Nucl. Instrum. Methods Phys. Res., Sect. A 515, 394 (2003).

[9] X. Q. Yan, Ph.D. dissertation, Peking University, 2004, p. 65 .

[10] K.R. Crandall, Los Alamos Group AT-1 Memorandum AT-1-237, 1980. 